\title{
General Decay for the Degenerate Equation with a Memory Condition at the Boundary
}

\author{
Su-Young Shin and Jum-Ran Kang \\ Department of Mathematics, Dong-A University, Saha-Ku, Busan 604-714, Republic of Korea \\ Correspondence should be addressed to Jum-Ran Kang; pointegg@hanmail.net \\ Received 21 December 2012; Accepted 5 March 2013 \\ Academic Editor: Abdelaziz Rhandi
}

Copyright ( 2013 S.-Y. Shin and J.-R. Kang. This is an open access article distributed under the Creative Commons Attribution License, which permits unrestricted use, distribution, and reproduction in any medium, provided the original work is properly cited.

We consider a degenerate equation with a memory condition at the boundary. For a wider class of relaxation functions, we establish a more general decay result, from which the usual exponential and polynomial decay rates are only special cases.

\section{Introduction}

The main purpose of this paper is to investigate the asymptotic behavior of the solutions of the degenerate equation with a memory condition at the boundary

$$
\begin{gathered}
K(x) u^{\prime \prime}+\Delta^{2} u+f(u)=0 \quad \text { in } Q=\Omega \times(0, \infty), \\
u=\frac{\partial u}{\partial v}=0 \quad \text { on } \Gamma_{0} \times(0, \infty), \\
-u+\int_{0}^{t} g_{1}(t-s) \mathscr{B}_{2} u(s) d s=0 \quad \text { on } \Gamma_{1} \times(0, \infty), \\
\frac{\partial u}{\partial v}+\int_{0}^{t} g_{2}(t-s) \mathscr{B}_{1} u(s) d s=0 \quad \text { on } \Gamma_{1} \times(0, \infty), \\
u(0, x)=u_{0}(x), \quad u^{\prime}(0, x)=u_{1}(x) \text { in } \Omega,
\end{gathered}
$$

where $\Omega$ is a bounded domain of $\mathbb{R}^{n}$ with a smooth boundary $\Gamma$ and let us assume that $\Gamma$, can be divided into two nonnull parts $\Gamma=\Gamma_{0} \cup \Gamma_{1}$ and $\overline{\Gamma_{0}} \cap \overline{\Gamma_{1}}=\emptyset$ and $K \in C^{1}(\bar{\Omega})$ and $K(x) \geq 0$ for all $x \in \Omega$ which satisfies some appropriate conditions. $\nu$ is the unit outward normal to $\Gamma$ and $\tau=\left(-v_{2}, v_{1}\right)$ the corresponding unit tangent vector. Here, the relaxation functions $g_{i}(i=1,2)$ are positive and nondecreasing, the function $f \in C^{1}(\mathbb{R})$ and

$$
\begin{gathered}
\mathscr{B}_{1} u=\Delta u+(1-\mu) B_{1} u, \quad \mathscr{B}_{2} u=\frac{\partial \Delta u}{\partial \nu}+(1-\mu) \frac{\partial B_{2} u}{\partial \tau}, \\
B_{1} u=2 v_{1} v_{2} u_{x y}-v_{1}^{2} u_{y y}-v_{2}^{2} u_{x x}, \\
B_{2} u=\left(v_{1}^{2}-v_{2}^{2}\right) u_{x y}+v_{1} v_{2}\left(u_{y y}-u_{x x}\right),
\end{gathered}
$$

and the constant $\mu, 0<\mu<1 / 2$, represents Poisson's ratio.

From the physical point of view, we know that the memory effect described in integral equations (3) and (4) can be caused by the interaction with another viscoelastic element. In fact, the boundary conditions (3) and (4) mean that $\Omega$ is composed of a material which is clamped in a rigid body in $\Gamma_{0}$ and is clamped in a body with viscoelastic properties in the complementary part of its boundary named $\Gamma_{1}$. Problems related to (1)-(5) are interesting not only from the point of view of PDE general theory, but also due to its applications in mechanics.

The existence of global solutions and exponential decay to the degenerate equation with $\partial \Omega=\Gamma_{0}$ has been investigated by several authors. See Cavalcanti et al. [1] and Menezes et al. [2]. For instance, when $K(x)$ is equal to 1, (1) describes the transverse deflection $u(x, t)$ of beams. There exists a large body of literature regarding viscoelastic problems with the memory term acting in the domain or at the boundary 
(see [3-17]). Santos et al. [18] studied the asymptotic behavior of the solutions of a nonlinear wave equation of Kirchhoff type with boundary condition of memory type. Cavalcanti et al. [19] proved the uniform decay rates of solutions to a degenerate system with a memory condition at the boundary. Santos and Junior [20] investigated the stability of solutions for Kirchhoff plate equations with a boundary memory condition. Rivera et al. [21] showed the asymptotic behavior to a von Karman plate with boundary memory conditions. Park and Kang [22] studied the exponential decay for the Kirchhoff plate equations with nonlinear dissipation and boundary memory condition. They proved that the energy decays uniformly exponentially or algebraically with the same rate of decay as the relaxation functions. In the present work, we generalize the earlier decay results of the solution of (1)-(5). More precisely, we show that the energy decays at the rate similar to the relaxation functions, which are not necessarily decaying like polynomial or exponential functions. In fact, our result allows a larger class of relaxation functions. Recently, Messaoudi and Soufyane [23], Mustafa and Messaoudi [24], and Santos and Soufyane [25] proved the general decay for the wave equation, Timoshenko system, and von Karman plate system with viscoelastic boundary conditions, respectively.

The organization of this paper is as follows. In Section 2, we present some notations and material needed for our work and state the existence result to system (1)-(5). In Section 3, we prove the general decay of the solutions to the degenerate equation with a memory condition at the boundary.

\section{Preliminaries}

In this section, we introduce some notations and establish the existence of solutions of the problem (1)-(5).

Note that, because of condition (2), the solution of system (1)-(5) must belong to the following space:

$$
W=\left\{v \in H^{2}(\Omega): v=\frac{\partial v}{\partial \nu}=0 \text { on } \Gamma_{0}\right\} .
$$

Let us define the bilinear form $a(\cdot, \cdot)$ as follows:

$$
\begin{gathered}
a(u, v)=\int_{\Omega}\left\{u_{x x} v_{x x}+u_{y y} v_{y y}+\mu\left(u_{x x} v_{y y}+u_{y y} v_{x x}\right)\right. \\
\left.+2(1-\mu) u_{x y} v_{x y}\right\} d x d y
\end{gathered}
$$

Since $\Gamma_{0} \neq \emptyset$, we know that $a(u, u)$ is equivalent to the $H^{2}(\Omega)$ norm on $W$; that is,

$$
c_{0}\|u\|_{H^{2}(\Omega)}^{2} \leq a(u, u) \leq C_{0}\|u\|_{H^{2}(\Omega)}^{2},
$$

and here and in the sequel, we denote by $c_{0}$ and $C_{0}$ generic positive constants. Simple calculation, based on the integration by parts formula, yields

$$
\left(\Delta^{2} u, v\right)=a(u, v)+\left(\mathscr{B}_{2} u, v\right)_{\Gamma}-\left(\mathscr{B}_{1} u, \frac{\partial v}{\partial v}\right)_{\Gamma}
$$

We assume that there exists $x_{0} \in \mathbb{R}^{n}$ such that

$$
\begin{aligned}
& \Gamma_{0}=\left\{x \in \Gamma: \nu(x) \cdot\left(x-x_{0}\right) \leq 0\right\}, \\
& \Gamma_{1}=\left\{x \in \Gamma: \nu(x) \cdot\left(x-x_{0}\right)>0\right\} .
\end{aligned}
$$

If we denote the compactness of $\Gamma_{1}$ by $m(x)=x-x_{0}$, condition (12) implies that there exists a small positive constant $\delta_{0}$ such that $0<\delta_{0} \leq m(x) \cdot \nu(x)$, for all $x \in \Gamma_{1}$.

Next, we will use (3) and (4) to estimate the values $\mathscr{B}_{1}$ and $\mathscr{B}_{2}$ on $\Gamma_{1}$. Denoting by

$$
(g * \varphi)(t)=\int_{0}^{t} g(t-s) \varphi(s) d s
$$

the convolution product operator and differentiating (3) and (4), we arrive at the following Volterra equations:

$$
\begin{gathered}
\mathscr{B}_{2} u+\frac{1}{g_{1}(0)} g_{1}^{\prime} * \mathscr{B}_{2} u=\frac{1}{g_{1}(0)} u^{\prime}, \\
\mathscr{B}_{1} u+\frac{1}{g_{2}(0)} g_{2}^{\prime} * \mathscr{B}_{1} u=-\frac{1}{g_{2}(0)} \frac{\partial u^{\prime}}{\partial v} .
\end{gathered}
$$

Applying the Volterra inverse operator, we get

$$
\begin{gathered}
\mathscr{B}_{2} u=\frac{1}{g_{1}(0)}\left\{u^{\prime}+k_{1} * u^{\prime}\right\}, \\
\mathscr{B}_{1} u=-\frac{1}{g_{2}(0)}\left\{\frac{\partial u^{\prime}}{\partial \nu}+k_{2} * \frac{\partial u^{\prime}}{\partial \nu}\right\},
\end{gathered}
$$

where the resolvent kernels satisfy

$$
k_{i}+\frac{1}{g_{i}(0)} g_{i}^{\prime} * k_{i}=-\frac{1}{g_{i}(0)} g_{i}^{\prime}, \quad \forall i=1,2 .
$$

Denoting that $\tau_{1}=1 / g_{1}(0)$ and $\tau_{2}=1 / g_{2}(0)$, we have

$$
\begin{gathered}
\mathscr{B}_{2} u=\tau_{1}\left\{u^{\prime}+k_{1}(0) u-k_{1}(t) u_{0}+k_{1}^{\prime} * u\right\}, \\
\mathscr{B}_{1} u=-\tau_{2}\left\{\frac{\partial u^{\prime}}{\partial \nu}+k_{2}(0) \frac{\partial u}{\partial \nu}-k_{2}(t) \frac{\partial u_{0}}{\partial \nu}+k_{2}^{\prime} * \frac{\partial u}{\partial \nu}\right\} .
\end{gathered}
$$

Therefore, we use (17) instead of the boundary conditions (3) and (4).

Let us denote that

$$
(g \diamond v)(t):=\int_{0}^{t} g(t-s)|v(t)-v(s)|^{2} d s .
$$

The following lemma states an important property of the convolution operator.

Lemma 1. For $g, v \in C^{1}([0, \infty): \mathbb{R})$, one has

$$
\begin{aligned}
(g * v) v^{\prime}= & -\frac{1}{2} g(t)|v(t)|^{2}+\frac{1}{2} g^{\prime} \diamond v-\frac{1}{2} \frac{d}{d t} \\
& \times\left[g \diamond v-\left(\int_{0}^{t} g(s) d s\right)|v|^{2}\right] .
\end{aligned}
$$


The proof of this lemma follows by differentiating the term $g \diamond v$.

Lemma 2 (see [26]). Suppose that $f \in L^{2}(\Omega), g \in H^{1 / 2}\left(\Gamma_{1}\right)$ and $h \in H^{3 / 2}\left(\Gamma_{1}\right)$; then, any solution of

$$
a(v, w)=\int_{\Omega} f w d x+\int_{\Gamma_{1}} g w d \Gamma+\int_{\Gamma_{1}} h \frac{\partial w}{\partial \nu} d \Gamma, \quad \forall w \in W
$$

satisfies $v \in H^{4}(\Omega)$ and also

$$
\begin{array}{ccc}
\Delta^{2} v=f, & v=\frac{\partial v}{\partial v}=0 & \text { on } \Gamma_{0}, \\
\mathscr{B}_{1} v=h, & \mathscr{B}_{2} v=g \quad \text { on } \Gamma_{1} .
\end{array}
$$

We formulate the following assumptions.

(A1) Let $f \in C^{1}(\mathbb{R})$ satisfy

$$
f(s) s \geq 0, \quad \forall s \in \mathbb{R} .
$$

Additionally, we suppose that $f$ is superlinear; that is,

$$
f(s) s \geq(2+\eta) F(s), \quad F(z)=\int_{0}^{z} f(s) d s, \quad \forall s \in \mathbb{R}
$$

for some $\eta>0$ with the following growth condition:

$$
|f(x)-f(y)| \leq c\left(1+|x|^{\rho-1}+|y|^{\rho-1}\right)|x-y|, \quad \forall x, y \in \mathbb{R}
$$

for some $c>0$ and $\rho \geq 1$ such that $(n-2) \rho \leq n$.

(A2) $K \in C^{1}(\bar{\Omega}) ; H_{0}^{2}(\Omega) \cap L^{\infty}(\Omega)$ with $K(x) \geq 0$, for all $x \in \Omega$, and satisfy the following condition

$$
\nabla K \cdot m \geq 0 \quad \text { in } \Omega \text {. }
$$

The well-posedness of system (1)-(5) is given by the following theorem.

Theorem 3 (see [27]). Consider assumptions (A1)-(A2) and let $k_{i} \in C^{2}\left(\mathbb{R}^{+}\right)$be such that

$$
k_{i},-k_{i}^{\prime}, k_{i}^{\prime \prime} \geq 0 \quad(i=1,2) .
$$

If $u_{0} \in W \cap H^{4}(\Omega), u_{1} \in W$, satisfying the compatibility condition

$$
\mathscr{B}_{1} u_{0}=-\tau_{2} \frac{\partial u_{1}}{\partial \nu}, \quad \mathscr{B}_{2} u_{0}=\tau_{1} u_{1} \quad \text { on } \Gamma_{1},
$$

then there is only one solution $u$ of the system (1)-(5) satisfying

$$
\begin{gathered}
u \in L^{\infty}\left(0, T: W \cap H^{4}(\Omega)\right), \quad u^{\prime} \in L^{\infty}(0, T: W), \\
u^{\prime \prime} \in L^{\infty}\left(0, T: L^{2}(\Omega)\right) .
\end{gathered}
$$

\section{General Decay}

In this section, we show that the solution of system (1)(5) may have a general decay not necessarily of exponential or polynomial type. For this we consider that the resolvent kernels satisfy the following hypothesis. that

(H) $k_{i}: \mathbb{R}_{+} \rightarrow \mathbb{R}_{+}$is twice differentiable function such

$$
k_{i}(0)>0, \quad \lim _{t \rightarrow \infty} k_{i}(t)=0, \quad k_{i}^{\prime}(t) \leq 0,
$$

and there exists a nonincreasing continuous function $\xi_{i}$ : $\mathbb{R}_{+} \rightarrow \mathbb{R}_{+}$satisfying

$$
k_{i}^{\prime \prime}(t) \geq-\xi_{i}(t) k_{i}^{\prime}(t), \quad i=1,2, \forall t \geq 0 .
$$

The following identity will be used later.

Lemma 4 (see [26]). For every $v \in H^{4}(\Omega)$ and for every $\mu \epsilon$ $\mathbb{R}$, one has

$$
\begin{aligned}
& \int_{\Omega}(m \cdot \nabla v) \Delta^{2} v d x \\
&= a(v, v) \\
&+\frac{1}{2} \int_{\Gamma} m \cdot v\left[v_{x x}^{2}+v_{y y}^{2}+2 \mu v_{x x} v_{y y}+2(1-\mu) v_{x y}^{2}\right] d \Gamma \\
&+\int_{\Gamma}\left[\left(\mathscr{B}_{2} v\right) m \cdot \nabla v-\left(\mathscr{B}_{1} v\right) \frac{\partial}{\partial v}(m \cdot \nabla v)\right] d \Gamma .
\end{aligned}
$$

Let us introduce the energy function

$$
\begin{aligned}
E(t)= & \frac{1}{2} \int_{\Omega} K(x)\left|u^{\prime}\right|^{2} d x+\frac{1}{2} a(u, u) \\
& +\int_{\Omega} F(u) d x+\frac{\tau_{1}}{2} \int_{\Gamma_{1}}\left(k_{1}(t)|u|^{2}-k_{1}^{\prime} \diamond u\right) d \Gamma \\
& +\frac{\tau_{2}}{2} \int_{\Gamma_{1}}\left(k_{2}(t)\left|\frac{\partial u}{\partial \nu}\right|^{2}-k_{2}^{\prime} \diamond \frac{\partial u}{\partial \nu}\right) d \Gamma
\end{aligned}
$$

Now, we establish some inequalities for the strong solution of system (1)-(5).

Lemma 5. The energy functional E satisfies, along the solution of (1)-(5), the estimate

$$
\begin{gathered}
E^{\prime}(t) \leq-\frac{\tau_{1}}{2} \int_{\Gamma_{1}}\left(\left|u^{\prime}\right|^{2}-k_{1}^{2}(t)\left|u_{0}\right|^{2}-k_{1}^{\prime}(t)|u|^{2}+k_{1}^{\prime \prime} \diamond u\right) d \Gamma \\
-\frac{\tau_{2}}{2} \int_{\Gamma_{1}}\left(\left|\frac{\partial u^{\prime}}{\partial \nu}\right|^{2}-k_{2}^{2}(t)\left|\frac{\partial u_{0}}{\partial \nu}\right|^{2}\right. \\
\left.-k_{2}^{\prime}(t)\left|\frac{\partial u}{\partial \nu}\right|^{2}+k_{2}^{\prime \prime} \diamond \frac{\partial u}{\partial \nu}\right) d \Gamma .
\end{gathered}
$$


Proof. Multiplying (1) by $u^{\prime}$, integrating over $\Omega$, and using (10), we get

$$
\begin{array}{r}
\frac{1}{2} \frac{d}{d t}\left\{\int_{\Omega} K\left|u^{\prime}\right|^{2} d x+a(u, u)+2 \int_{\Omega} F(u) d x\right\} \\
=-\int_{\Gamma_{1}}\left(\mathscr{B}_{2} u\right) u^{\prime} d \Gamma+\int_{\Gamma_{1}}\left(\mathscr{B}_{1} u\right) \frac{\partial u^{\prime}}{\partial \nu} d \Gamma
\end{array}
$$

Substituting the boundary terms by (17) and using Lemma 1 and the Young inequality, our conclusion follows.

Let us consider the following binary operator:

$$
(k \circ u)(t):=\int_{0}^{t} k(t-s)(u(t)-u(s)) d s .
$$

Then applying the Holder inequality for $0 \leq \alpha \leq 1$ we have

$$
|(k \circ u)(t)|^{2} \leq\left[\int_{0}^{t}|k(s)|^{2(1-\alpha)} d s\right]\left(|k|^{2 \alpha} \diamond u\right)(t) .
$$

Let us define the functional

$$
\psi(t)=\int_{\Omega}\left[m \cdot \nabla u+\left(\frac{n}{2}-\theta\right) u\right] K u^{\prime} d x
$$

The following lemma plays an important role in the construction of the desired functional.

Lemma 6. Suppose that the initial data $\left(u_{0}, u_{1}\right) \in\left(H^{4}(\Omega) \cap\right.$ $W) \times W$, satisfying the compatibility condition (27). Then, the solution of system (1)-(5) satisfies

$$
\begin{aligned}
\psi^{\prime}(t) \leq & \frac{1}{2} \int_{\Gamma_{1}} m \cdot v K\left|u^{\prime}\right|^{2} d \Gamma \\
& -\theta \int_{\Omega} K\left|u^{\prime}\right|^{2} d x-\left(1+\frac{n}{2}-\theta-\epsilon \lambda_{0}\right) a(u, u) \\
& -\left(\frac{n \eta}{2}-2 \theta-\eta \theta\right) \int_{\Omega} F(u) d x+\frac{2 \tau_{1}^{2}}{\epsilon} \\
& \times \int_{\Gamma_{1}}\left\{\left|u^{\prime}\right|^{2}+k_{1}^{2}(t)|u|^{2}+k_{1}^{2}(t)\left|u_{0}\right|^{2}+\left|k_{1}^{\prime} \circ u\right|^{2}\right\} d \Gamma \\
& +\frac{2 \tau_{2}^{2}}{\epsilon} \int_{\Gamma_{1}}\left\{\left|\frac{\partial u^{\prime}}{\partial \nu}\right|^{2}+k_{2}^{2}(t)\left|\frac{\partial u}{\partial \nu}\right|^{2}+k_{2}^{2}(t)\left|\frac{\partial u_{0}}{\partial \nu}\right|^{2}\right. \\
& -\left(\frac{1}{2}-\frac{\epsilon \lambda_{0}}{\delta_{0}}\right) \int_{\Gamma_{1}} m \cdot v\left[\left.k_{x x}^{\prime} \circ \frac{\partial u}{\partial \nu}\right|^{2}\right\} d \Gamma \\
& \left.+2(1-\mu) u_{x y}^{2}\right] d \Gamma .2 \mu u_{x x} u_{y y}
\end{aligned}
$$

Proof. Differentiating $\psi$ using (1) and Lemma 4, we get

$$
\begin{aligned}
\psi^{\prime}(t)= & \int_{\Omega}\left[m \cdot \nabla u^{\prime}+\left(\frac{n}{2}-\theta\right) u^{\prime}\right] K u^{\prime} d x \\
& +\int_{\Omega}\left[m \cdot \nabla u+\left(\frac{n}{2}-\theta\right) u\right] K u^{\prime \prime} d x \\
= & \frac{1}{2} \int_{\Gamma_{1}} m \cdot v K\left|u^{\prime}\right|^{2} d \Gamma-\theta \int_{\Omega} K\left|u^{\prime}\right|^{2} d x \\
& -\frac{1}{2} \int_{\Omega} \nabla K \cdot m\left|u^{\prime}\right|^{2} d x-\left(1+\frac{n}{2}-\theta\right) a(u, u) \\
& +n \int_{\Omega} F(u) d x-\left(\frac{n}{2}-\theta\right) \int_{\Omega} f(u) u d x \\
& -\frac{1}{2} \int_{\Gamma} m \cdot v\left[u_{x x}^{2}+u_{y y}^{2}+2 \mu u_{x x} u_{y y}+2(1-\mu) u_{x y}^{2}\right] d \Gamma \\
& -\int_{\Gamma}\left(\mathscr{B}_{2} u\right)\left[(m \cdot \nabla u)+\left(\frac{n}{2}-\theta\right) u\right] d \Gamma \\
& +\int_{\Gamma}\left(\mathscr{B}_{1} u\right)\left[\frac{\partial}{\partial v}(m \cdot \nabla u)+\left(\frac{n}{2}-\theta\right) \frac{\partial u}{\partial v}\right] d \Gamma .
\end{aligned}
$$

Let us next examine the integrals over $\Gamma_{0}$ in (39). Since $u=$ $\partial u / \partial v=0$ on $\Gamma_{0}$, we have $B_{1} u=B_{2} u=0$ on $\Gamma_{0}$ and

$$
\begin{gathered}
\frac{\partial}{\partial \nu}(m \cdot \nabla u)=(m \cdot \nu) \Delta u \\
u_{x x}^{2}+u_{y y}^{2}+2 \mu u_{x x} u_{y y}+2(1-\mu) u_{x y}^{2}=(\Delta u)^{2} \text { on } \Gamma_{0},
\end{gathered}
$$

since

$$
u_{x x} u_{y y}-u_{x y}^{2}=0 \text { on } \Gamma_{0} \text {. }
$$

Therefore, from (39) and (40), we have

$$
\begin{aligned}
\psi^{\prime}(t) \leq & \frac{1}{2} \int_{\Gamma_{1}} m \cdot v K\left|u^{\prime}\right|^{2} d \Gamma-\theta \int_{\Omega} K\left|u^{\prime}\right|^{2} d x \\
& -\frac{1}{2} \int_{\Omega} \nabla K \cdot m\left|u^{\prime}\right|^{2} d x-\left(1+\frac{n}{2}-\theta\right) a(u, u) \\
& +n \int_{\Omega} F(u) d x-\left(\frac{n}{2}-\theta\right) \int_{\Omega} f(u) u d x \\
& +\frac{1}{2} \int_{\Gamma_{0}} m \cdot v(\Delta u)^{2} d \Gamma \\
& -\frac{1}{2} \int_{\Gamma_{1}} m \cdot v\left[u_{x x}^{2}+u_{y y}^{2}+2 \mu u_{x x} u_{y y}+2(1-\mu) u_{x y}^{2}\right] d \Gamma \\
& -\int_{\Gamma_{1}}\left(\mathscr{B}_{2} u\right)\left[(m \cdot \nabla u)+\left(\frac{n}{2}-\theta\right) u\right] d \Gamma \\
& +\int_{\Gamma_{1}}\left(\mathscr{B}_{1} u\right)\left[\frac{\partial}{\partial v}(m \cdot \nabla u)+\left(\frac{n}{2}-\theta\right) \frac{\partial u}{\partial v}\right] d \Gamma .
\end{aligned}
$$


Using the Young inequality, we get

$$
\begin{aligned}
& \left|\int_{\Gamma_{1}}\left(\mathscr{B}_{2} u\right)\left[(m \cdot \nabla u)+\left(\frac{n}{2}-\theta\right) u\right] d \Gamma\right| \\
& \quad \leq \frac{1}{2 \epsilon} \int_{\Gamma_{1}}\left|\mathscr{B}_{2} u\right|^{2} d \Gamma+\epsilon \int_{\Gamma_{1}}\left(|m \cdot \nabla u|^{2}+\left(\frac{n}{2}-\theta\right)^{2}|u|^{2}\right) d \Gamma,
\end{aligned}
$$

$$
\begin{aligned}
& \left|\int_{\Gamma_{1}}\left(\mathscr{B}_{1} u\right)\left[\frac{\partial}{\partial \nu}(m \cdot \nabla u)+\left(\frac{n}{2}-\theta\right) \frac{\partial u}{\partial \nu}\right] d \Gamma\right| \\
& \leq \frac{1}{2 \epsilon} \int_{\Gamma_{1}}\left|\mathscr{B}_{1} u\right|^{2} d \Gamma \\
& \quad+\epsilon \int_{\Gamma_{1}}\left(\left|\frac{\partial}{\partial \nu}(m \cdot \nabla u)\right|^{2}+\left(\frac{n}{2}-\theta\right)^{2}\left|\frac{\partial u}{\partial \nu}\right|^{2}\right) d \Gamma
\end{aligned}
$$

where $\epsilon$ is a positive constant. Since the bilinear form $a(u, u)$ is strictly coercive on $W$, using the trace theory, we obtain

$$
\begin{aligned}
\int_{\Gamma_{1}}\left(|m \cdot \nabla u|^{2}+\left(\frac{n}{2}-\theta\right)^{2}|u|^{2}\right) d \Gamma \\
\quad+\int_{\Gamma_{1}}\left(\left|\frac{\partial}{\partial \nu}(m \cdot \nabla u)\right|^{2}+\left(\frac{n}{2}-\theta\right)^{2}\left|\frac{\partial u}{\partial \nu}\right|^{2}\right) d \Gamma \\
\leq \lambda_{0} a(u, u)+\frac{\lambda_{0}}{\delta_{0}} \\
\quad \times \int_{\Gamma_{1}} m \cdot v\left[u_{x x}^{2}+u_{y y}^{2}+2 \mu u_{x x} u_{y y}+2(1-\mu) u_{x y}^{2}\right] d \Gamma
\end{aligned}
$$

where $\lambda_{0}$ is a constant depending on $\Omega, \mu, \theta$ and $n$. Substituting inequalities (43)-(45) into (42) and taking into account that $m \cdot v \leq 0$ on $\Gamma_{0}$, as well as (23) and (25), we have

$$
\begin{aligned}
\psi^{\prime}(t) \leq & \frac{1}{2} \int_{\Gamma_{1}} m \cdot v K\left|u^{\prime}\right|^{2} d \Gamma-\theta \int_{\Omega} K\left|u^{\prime}\right|^{2} d x \\
& -\left(1+\frac{n}{2}-\theta-\epsilon \lambda_{0}\right) a(u, u) \\
& -\left(\frac{n \eta}{2}-2 \theta-\eta \theta\right) \int_{\Omega} F(u) d x \\
& +\frac{1}{2 \epsilon} \int_{\Gamma_{1}}\left(\left|\mathscr{B}_{1} u\right|^{2}+\left|\mathscr{B}_{2} u\right|^{2}\right) d \Gamma \\
& -\left(\frac{1}{2}-\frac{\epsilon \lambda_{0}}{\delta_{0}}\right) \int_{\Gamma_{1}} m \cdot v\left[u_{x x}^{2}+u_{y y}^{2}+2 \mu u_{x x} u_{y y}\right. \\
& \left.+2(1-\mu) u_{x y}^{2}\right] d \Gamma .
\end{aligned}
$$

Since the boundary conditions (17) can be written as

$$
\mathscr{B}_{2} u=\tau_{1}\left\{u^{\prime}+k_{1}(t) u-k_{1}(t) u_{0}-k_{1}^{\prime} \circ u\right\},
$$

$$
\mathscr{B}_{1} u=-\tau_{2}\left\{\frac{\partial u^{\prime}}{\partial \nu}+k_{2}(t) \frac{\partial u}{\partial \nu}-k_{2}(t) \frac{\partial u_{0}}{\partial \nu}-k_{2}^{\prime} \circ \frac{\partial u}{\partial \nu}\right\}
$$

our conclusion follows.

Let us introduce the Lyapunov functional

$$
\mathscr{L}(t)=N E(t)+\psi(t),
$$

with $N>0$. Now, we are in a position to show the main result of this paper.

Theorem 7. Let $\left(u_{0}, u_{1}\right) \in W \times L^{2}(\Omega)$. Suppose that the resolvent kernels $k_{1}, k_{2}$ satisfy the condition $(H)$. Then, there exist constants $\omega, C>0$ such that, for some $t_{0}$ large enough, the solution of (1)-(5) satisfies

$E(t) \leq C E(0) e^{-\omega \int_{0}^{t} \xi(s) d s}, \quad \forall t \geq t_{0}$, if $u_{0}=\frac{\partial u_{0}}{\partial v}=0 \quad$ on $\Gamma_{1}$.

Otherwise,

$$
E(t) \leq C\left(E(0)+\int_{0}^{t} k_{0}(s) e^{\omega \int_{t_{0}}^{s} \xi(\tau) d \tau} d s\right) e^{-\omega \int_{0}^{t} \xi(s) d s}
$$

for all $t \geq t_{0}$, where

$$
\begin{gathered}
\xi(t)=\min \left\{\xi_{1}(t), \xi_{2}(t)\right\} \\
k_{0}(t)=\int_{\Gamma_{1}} k_{1}^{2}(t)\left|u_{0}\right|^{2} d \Gamma+\int_{\Gamma_{1}} k_{2}^{2}(t)\left|\frac{\partial u_{0}}{\partial \nu}\right|^{2} d \Gamma .
\end{gathered}
$$

Proof. Applying inequality (36) with $\alpha=1 / 2$ in Lemma 6 and from Lemma 5, we obtain

$$
\begin{aligned}
\mathscr{L}^{\prime}(t) \leq & -\int_{\Omega} \theta K\left|u^{\prime}\right|^{2} d x \\
& -\frac{\tau_{1} N}{2} \int_{\Gamma_{1}}\left\{\left|u^{\prime}\right|^{2}-k_{1}^{2}(t)\left|u_{0}\right|^{2}-k_{1}^{\prime}(t)|u|^{2}+k_{1}^{\prime \prime} \diamond u\right\} d \Gamma \\
& -\frac{\tau_{2} N}{2} \int_{\Gamma_{1}}\left\{\left|\frac{\partial u^{\prime}}{\partial \nu}\right|^{2}-k_{2}^{2}(t)\left|\frac{\partial u_{0}}{\partial \nu}\right|^{2}-k_{2}^{\prime}(t)\left|\frac{\partial u}{\partial \nu}\right|^{2}\right. \\
& \left.+k_{2}^{\prime \prime} \diamond \frac{\partial u}{\partial \nu}\right\} d \Gamma \\
& -\left(1+\frac{n}{2}-\theta-\epsilon \lambda_{0}\right) a(u, u)-\left(\frac{n \eta}{2}-2 \theta-\eta \theta\right) \\
& \times \int_{\Omega} F(u) d x+\frac{2 \tau_{1}^{2}}{\epsilon} \int_{\Gamma_{1}}\left\{\left|u^{\prime}\right|^{2}+k_{1}^{2}(t)|u|^{2}+k_{1}^{2}(t)\left|u_{0}\right|^{2}\right. \\
& \left.-k_{1}(0) k_{1}^{\prime} \diamond u\right\} d \Gamma
\end{aligned}
$$




$$
\begin{aligned}
& +\frac{2 \tau_{2}^{2}}{\epsilon} \int_{\Gamma_{1}}\left\{\left|\frac{\partial u^{\prime}}{\partial \nu}\right|^{2}+k_{2}^{2}(t)\left|\frac{\partial u}{\partial \nu}\right|^{2}+k_{2}^{2}(t)\left|\frac{\partial u_{0}}{\partial \nu}\right|^{2}\right. \\
& \left.\quad-k_{2}(0) k_{2}^{\prime} \diamond \frac{\partial u}{\partial \nu}\right\} d \Gamma \\
& +\frac{1}{2} \int_{\Gamma_{1}} m \cdot v K\left|u^{\prime}\right|^{2} d \Gamma-\left(\frac{1}{2}-\frac{\epsilon \lambda_{0}}{\delta_{0}}\right) \\
& \times \int_{\Gamma_{1}} m \cdot v\left[u_{x x}^{2}+u_{y y}^{2}+2 \mu u_{x x} u_{y y}+2(1-\mu) u_{x y}^{2}\right] d \Gamma .
\end{aligned}
$$

We take $\theta$ and $\epsilon$ so small such that

$$
\begin{aligned}
& \frac{n \eta}{2}-2 \theta-\eta \theta> 0, \quad 1+\frac{n}{2}-\theta-\epsilon \lambda_{0}>0, \\
& \frac{1}{2}-\frac{\epsilon \lambda_{0}}{\delta_{0}}>0 .
\end{aligned}
$$

Since $K \in L^{\infty}(\Omega)$ and then choosing $N$ large enough, we obtain

$$
\begin{aligned}
\mathscr{L}^{\prime}(t) \leq & -c_{0} E(t)+c \int_{\Gamma_{1}} k_{1}^{2}(t)\left|u_{0}\right|^{2} d \Gamma+c \int_{\Gamma_{1}} k_{2}^{2}(t)\left|\frac{\partial u_{0}}{\partial \nu}\right|^{2} d \Gamma \\
& -c \int_{\Gamma_{1}} k_{1}^{\prime} \diamond u d \Gamma-c \int_{\Gamma_{1}} k_{2}^{\prime} \diamond \frac{\partial u}{\partial \nu} d \Gamma, \quad \forall t \geq t_{0} .
\end{aligned}
$$

On the other hand, we can choose $N$ even larger so that

$$
\mathscr{L}(t) \sim E(t) .
$$

If $\xi(t)=\min \left\{\xi_{1}(t), \xi_{2}(t)\right\}, t \geq t_{0}$, then, using (30) and (33), we have

$$
\begin{aligned}
\xi(t) \mathscr{L}^{\prime}(t) \leq & -c_{0} \xi(t) E(t)+c \xi(t) \int_{\Gamma_{1}} k_{1}^{2}(t)\left|u_{0}\right|^{2} d \Gamma \\
& +c \xi(t) \int_{\Gamma_{1}} k_{2}^{2}(t)\left|\frac{\partial u_{0}}{\partial \nu}\right|^{2} d \Gamma \\
& -c \xi_{1}(t) \int_{\Gamma_{1}} k_{1}^{\prime} \diamond u d \Gamma-c \xi_{2}(t) \int_{\Gamma_{1}} k_{2}^{\prime} \diamond \frac{\partial u}{\partial \nu} d \Gamma \\
\leq & -c_{0} \xi(t) E(t)+c \xi(t) \int_{\Gamma_{1}} k_{1}^{2}(t)\left|u_{0}\right|^{2} d \Gamma \\
& +c \xi(t) \int_{\Gamma_{1}} k_{2}^{2}(t)\left|\frac{\partial u_{0}}{\partial \nu}\right|^{2} d \Gamma \\
& +c \int_{\Gamma_{1}} k_{1}^{\prime \prime} \diamond u d \Gamma+c \int_{\Gamma_{1}} k_{2}^{\prime \prime} \diamond \frac{\partial u}{\partial \nu} d \Gamma
\end{aligned}
$$

$$
\begin{aligned}
\leq & -c_{0} \xi(t) E(t)+c \xi(t) \int_{\Gamma_{1}} k_{1}^{2}(t)\left|u_{0}\right|^{2} d \Gamma \\
& +c \xi(t) \int_{\Gamma_{1}} k_{2}^{2}(t)\left|\frac{\partial u_{0}}{\partial \nu}\right|^{2} d \Gamma-c E^{\prime}(t), \quad \forall t \geq t_{0},
\end{aligned}
$$

which gives

$$
\begin{aligned}
\xi(t) \mathscr{L}^{\prime}(t)+c E^{\prime}(t) \leq & -c_{0} \xi(t) E(t)+c \xi(t) \int_{\Gamma_{1}} k_{1}^{2}(t)\left|u_{0}\right|^{2} d \Gamma \\
& +c \xi(t) \int_{\Gamma_{1}} k_{2}^{2}(t)\left|\frac{\partial u_{0}}{\partial \nu}\right|^{2} d \Gamma, \quad \forall t \geq t_{0} .
\end{aligned}
$$

Using the fact that $\xi$ is a nonincreasing continuous function as $\xi_{1}$ and $\xi_{2}$ are nonincreasing, and so $\xi$ is differentiable, with $\xi^{\prime}(t) \leq 0$, for a.e. $t$, then we infer that

$$
\begin{aligned}
(\xi \mathscr{L}+c E)^{\prime}(t) \leq & \xi(t) \mathscr{L}^{\prime}(t)+c E^{\prime}(t) \\
\leq & -c_{0} \xi(t) E(t)+c \xi(t) \int_{\Gamma_{1}} k_{1}^{2}(t)\left|u_{0}\right|^{2} d \Gamma \\
& +c \xi(t) \int_{\Gamma_{1}} k_{2}^{2}(t)\left|\frac{\partial u_{0}}{\partial \nu}\right|^{2} d \Gamma, \quad \forall t \geq t_{0} .
\end{aligned}
$$

Since using (55),

$$
F=\xi \mathscr{L}+c E \sim E
$$

we obtain, for some positive constant $\omega$,

$$
\begin{aligned}
F^{\prime}(t) \leq & -\omega \xi(t) F(t)+c \int_{\Gamma_{1}} k_{1}^{2}(t)\left|u_{0}\right|^{2} d \Gamma \\
& +c \int_{\Gamma_{1}} k_{2}^{2}(t)\left|\frac{\partial u_{0}}{\partial \nu}\right|^{2} d \Gamma, \quad \forall t \geq t_{0} .
\end{aligned}
$$

Case 1. If $u_{0}=\partial u_{0} / \partial v=0$ on $\Gamma_{1}$, then (60) reduces to

$$
F^{\prime}(t) \leq-\omega \xi(t) F(t), \quad \forall t \geq t_{0} .
$$

A simple integration over $\left(t_{0}, t\right)$ yields

$$
F(t) \leq F\left(t_{0}\right) e^{-\omega \int_{t_{0}}^{t} \xi(s) d s}, \quad \forall t \geq t_{0} .
$$

By using (33) and (59), we then obtain for some positive constant $C$

$$
E(t) \leq C E(0) e^{-\omega \int_{0}^{t} \xi(s) d s}, \quad \forall t \geq t_{0} .
$$

Thus, estimate (49) is proved.

Case 2. If $\left(u_{0},\left(\partial u_{0} / \partial \nu\right)\right) \neq(0,0)$ on $\Gamma_{1}$, then $(60)$ gives

$$
F^{\prime}(t) \leq-\omega \xi(t) F(t)+c k_{0}(t), \quad \forall t \geq t_{0}
$$


where

$$
k_{0}(t)=\int_{\Gamma_{1}} k_{1}^{2}(t)\left|u_{0}\right|^{2} d \Gamma+\int_{\Gamma_{1}} k_{2}^{2}(t)\left|\frac{\partial u_{0}}{\partial \nu}\right|^{2} d \Gamma
$$

In this case, we introduce

$$
G(t):=F(t)-c e^{-\omega \int_{t_{0}}^{t} \xi(s) d s} \int_{t_{0}}^{t} k_{0}(s) e^{\omega \int_{t_{0}}^{s} \xi(\tau) d \tau} d s .
$$

A simple differentiation of $G$, using (64), leads to

$$
\begin{aligned}
G^{\prime}(t)= & F^{\prime}(t)+\omega \xi(t) c e^{-\omega \int_{t_{0}}^{t} \xi(s) d s} \\
& \times \int_{t_{0}}^{t} k_{0}(s) e^{\omega \int_{t_{0}}^{s} \xi(\tau) d \tau} d s-c k_{0}(t) \\
\leq & -\omega \xi(t) G(t), \quad \forall t \geq t_{0} .
\end{aligned}
$$

Again, a simple integration over $\left(t_{0}, t\right)$ yields

$$
G(t) \leq G\left(t_{0}\right) e^{-\omega \int_{t_{0}}^{t} \xi(s) d s}, \quad \forall t \geq t_{0},
$$

which implies, for all $t \geq t_{0}$,

$$
F(t) \leq\left(F\left(t_{0}\right)+c \int_{t_{0}}^{t} k_{0}(s) e^{\omega \int_{t_{0}}^{s} \xi(\tau) d \tau} d s\right) e^{-\omega \int_{t_{0}}^{t} \xi(s) d s} .
$$

By using (59), we deduce that

$$
\begin{array}{r}
E(t) \leq C\left(E(0)+\int_{0}^{t} k_{0}(s) e^{\omega \int_{t_{0}}^{s} \xi(\tau) d \tau} d s\right) e^{-\omega \int_{t_{0}}^{t} \xi(s) d s}, \\
\forall t \geq t_{0} .
\end{array}
$$

Consequently, by the boundedness of $\xi$, (50) is established.

Remark 8. Note that the exponential and polynomial decay estimates are only particular cases of (49) and (50). More precisely, we have exponential decay for $\xi_{1}(t) \equiv c_{1}$ and $\xi_{2}(t) \equiv$ $c_{2}$ and polynomial decay for $\xi_{1}(t)=c_{1}(1+t)^{-1}$ and $\xi_{2}(t) \equiv c_{2}$, where $c_{1}$ and $c_{2}$ are positive constants.

Example 9. As in [24], we give some examples to illustrate the energy decay rates given by (49).

(1) If $k_{1}(t)=k_{2}(t)=a e^{-b(1+t)^{p}}, 0<p \leq 1$, then, for $i=$ $1,2, k_{i}^{\prime \prime}(t) \geq-\xi(t) k_{i}^{\prime}(t)$, where $\xi(t)=b p(1+t)^{p-1}$. For suitably chosen positive constants $a$ and $b, k_{i}$ satisfies $(\mathrm{H})$ and $(49)$ gives

$$
E(t) \leq c e^{-\omega b(1+t)^{p}}
$$

(2) If $k_{1}(t)=a_{1} /(1+t)^{q}, q>0$, and $k_{2}(t)=$ $a_{2} e^{-b(1+t)^{p}}, 0<p \leq 1$, then, for $i=1,2, k_{i}^{\prime \prime}(t) \geq$ $-\xi(t) k_{i}^{\prime}(t)$, where $\xi(t)=q(1+t)^{-1}$. Then

$$
E(t) \leq \frac{c}{(1+t)^{\omega q}} .
$$

The aforementioned two examples are included in the following more general one.
(3) For any nonincreasing functions $k_{i}(t), i=1,2$, which satisfy $(\mathrm{H}), \xi_{i}=-k^{\prime} / k$ are also nonincreasing differentiable functions, and $c \xi_{1}(t) \leq \xi_{2}(t)$, for some $0<c \leq 1$, and (49) gives

$$
E(t) \leq c\left[k_{1}(t)\right]^{\omega} .
$$

\section{Acknowledgment}

This research was supported by Basic Science Research Program through the National Research Foundation of Korea (NRF) funded by the Ministry of Education, Science and Technology (Grant no. 2012R1A1A3011630).

\section{References}

[1] M. M. Cavalcanti, V. N. Domingos Cavalcanti, J. S. Prates Filho, and J. A. Soriano, "Existence and uniform decay of solutions of a degenerate equation with nonlinear boundary damping and boundary memory source term," Nonlinear Analysis: Theory, Methods \& Applications, vol. 38, no. 3, pp. 281-294, 1999.

[2] S. D. B. Menezes, E. A. de Oliveira, D. C. Pereira, and J. Ferreira, "Existence, uniqueness and uniform decay for the nonlinear beam degenerate equation with weak damping," Applied Mathematics and Computation, vol. 154, no. 2, pp. 555-565, 2004.

[3] M. Aassila, M. M. Cavalcanti, and V. N. Domingos Cavalcanti, "Existence and uniform decay of the wave equation with nonlinear boundary damping and boundary memory source term," Calculus of Variations and Partial Differential Equations, vol. 15, no. 2, pp. 155-180, 2002.

[4] M. Aassila, M. M. Cavalcanti, and J. A. Soriano, "Asymptotic stability and energy decay rates for solutions of the wave equation with memory in a star-shaped domain," SIAM Journal on Control and Optimization, vol. 38, no. 5, pp. 1581-1602, 2000.

[5] F. Alabau-Boussouira, P. Cannarsa, and D. Sforza, "Decay estimates for second order evolution equations with memory," Journal of Functional Analysis, vol. 254, no. 5, pp. 1342-1372, 2008.

[6] F. Alabau-Boussouira, J. Prüss, and R. Zacher, "Exponential and polynomial stability of a wave equation for boundary memory damping with singular kernels," Comptes Rendus Mathematique, vol. 347, no. 5-6, pp. 277-282, 2009.

[7] M. M. Cavalcanti, V. N. Domingos Cavalcanti, and J. Ferreira, "Existence and uniform decay for a non-linear viscoelastic equation with strong damping," Mathematical Methods in the Applied Sciences, vol. 24, no. 14, pp. 1043-1053, 2001.

[8] M. M. Cavalcanti, V. N. Domingos Cavalcanti, and P. Martinez, "General decay rate estimates for viscoelastic dissipative systems," Nonlinear Analysis: Theory, Methods \& Applications, vol. 68, no. 1, pp. 177-193, 2008.

[9] M. M. Cavalcanti, V. N. Domingos Cavalcanti, J. S. Prates, and J. A. Soriano, "Existence and uniform decay rates for viscoelastic problems with nonlinear boundary damping," Differential and Integral Equations, vol. 14, no. 1, pp. 85-116, 2001.

[10] M. M. Cavalcanti and H. P. Oquendo, "Frictional versus viscoelastic damping in a semilinear wave equation," SIAM Journal on Control and Optimization, vol. 42, no. 4, pp. 1310-1324, 2003.

[11] A. Guesmia and S. A. Messaoudi, "General energy decay estimates of Timoshenko systems with frictional versus viscoelastic damping," Mathematical Methods in the Applied Sciences, vol. 32 , no. 16, pp. 2102-2122, 2009. 
[12] X. S. Han and M. X. Wang, "Global existence and uniform decay for a nonlinear viscoelastic equation with damping," Nonlinear Analysis: Theory, Methods \& Applications, vol. 70, no. 9, pp. 3090-3098, 2009.

[13] J. R. Kang, "Energy decay rates for von Kármán system with memory and boundary feedback," Applied Mathematics and Computation, vol. 218, no. 18, pp. 9085-9094, 2012.

[14] S. A. Messaoudi, "General decay of solutions of a viscoelastic equation," Journal of Mathematical Analysis and Applications, vol. 341, no. 2, pp. 1457-1467, 2008.

[15] S. A. Messaoudi, "General decay of the solution energy in a viscoelastic equation with a nonlinear source," Nonlinear Analysis: Theory, Methods \& Applications, vol. 69, no. 8, pp. 2589-2598, 2008.

[16] S. A. Messaoudi and M. I. Mustafa, "On convexity for energy decay rates of a viscoelastic equation with boundary feedback," Nonlinear Analysis: Theory, Methods \& Applications, vol. 72, no. 9-10, pp. 3602-3611, 2010.

[17] J. Prüss, "Decay properties for the solutions of a partial differential equation with memory," Archiv der Mathematik, vol. 92, no. 2, pp. 158-173, 2009.

[18] M. L. Santos, J. Ferreira, D. C. Pereira, and C. A. Raposo, "Global existence and stability for wave equation of Kirchhoff type with memory condition at the boundary," Nonlinear Analysis: Theory, Methods \& Applications, vol. 54, no. 5, pp. 959-976, 2003.

[19] M. M. Cavalcanti, V. N. Domingos Cavalcanti, and M. L. Santos, "Existence and uniform decay rates of solutions to a degenerate system with memory conditions at the boundary," Applied Mathematics and Computation, vol. 150, no. 2, pp. 439-465, 2004.

[20] M. L. Santos and F. Junior, "A boundary condition with memory for Kirchhoff plates equations," Applied Mathematics and Computation, vol. 148, no. 2, pp. 475-496, 2004.

[21] J. E. M. Rivera, H. P. Oquendo, and M. L. Santos, "Asymptotic behavior to a von Kármán plate with boundary memory conditions," Nonlinear Analysis: Theory, Methods \& Applications, vol. 62, no. 7, pp. 1183-1205, 2005.

[22] J. Y. Park and J. R. Kang, "Uniform decay for hyperbolic differential inclusion with memory condition at the boundary," Numerical Functional Analysis and Optimization, vol. 27, no. 78, pp. 875-888, 2006.

[23] S. A. Messaoudi and A. Soufyane, "General decay of solutions of a wave equation with a boundary control of memory type," Nonlinear Analysis: Real World Applications, vol. 11, no. 4, pp. 2896-2904, 2010.

[24] M. I. Mustafa and S. A. Messaoudi, "Energy decay rates for a Timoshenko system with viscoelastic boundary conditions," Applied Mathematics and Computation, vol. 218, no. 18, pp. 9125-9131, 2012.

[25] M. L. Santos and A. Soufyane, "General decay to a von Kármán plate system with memory boundary conditions," Differential and Integral Equations, vol. 24, no. 1-2, pp. 69-81, 2011.

[26] J. E. Lagnese, Boundary Stabilization of Thin Plates, SIAM. Society for Industrial and Applied Mathematics, Philadelphia, Pa, USA, 1989.

[27] J. Y. Park and J. R. Kang, "Existence, uniqueness and uniform decay for the non-linear degenerate equation with memory condition at the boundary," Applied Mathematics and Computation, vol. 202 , no. 2 , pp. 481-488, 2008. 


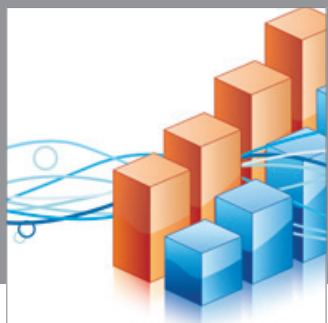

Advances in

Operations Research

mansans

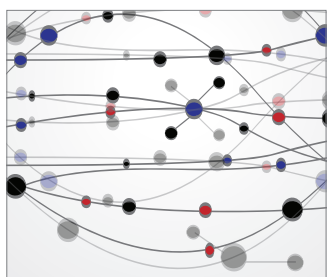

The Scientific World Journal
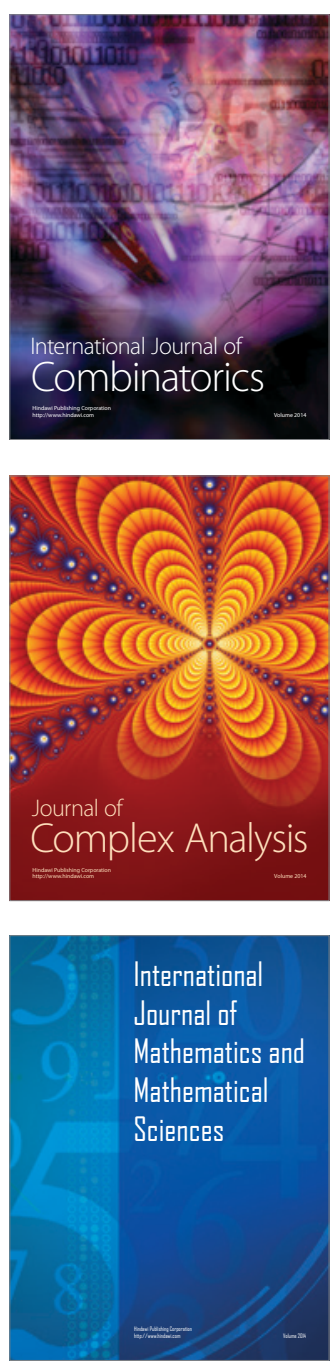
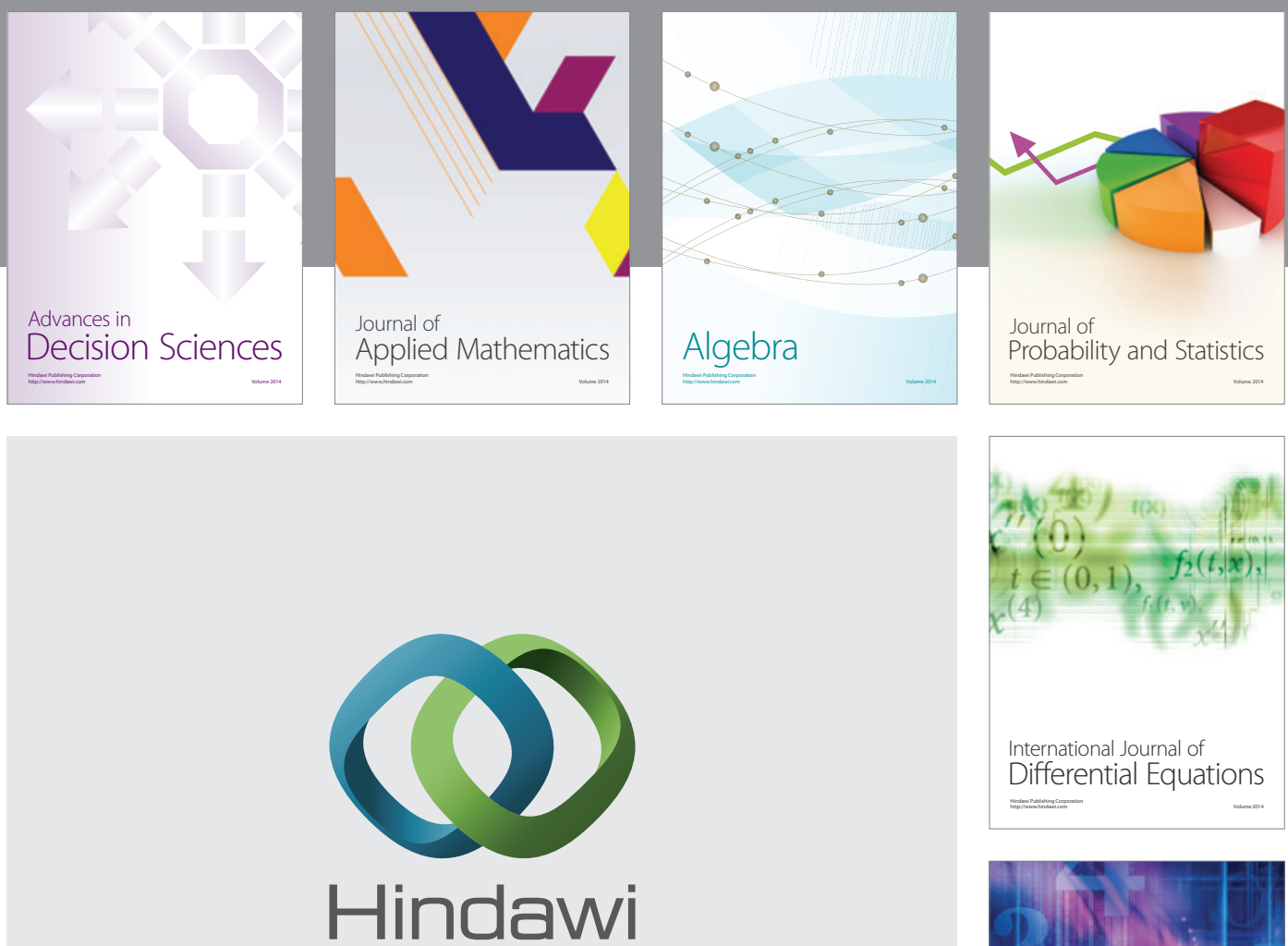

Submit your manuscripts at http://www.hindawi.com
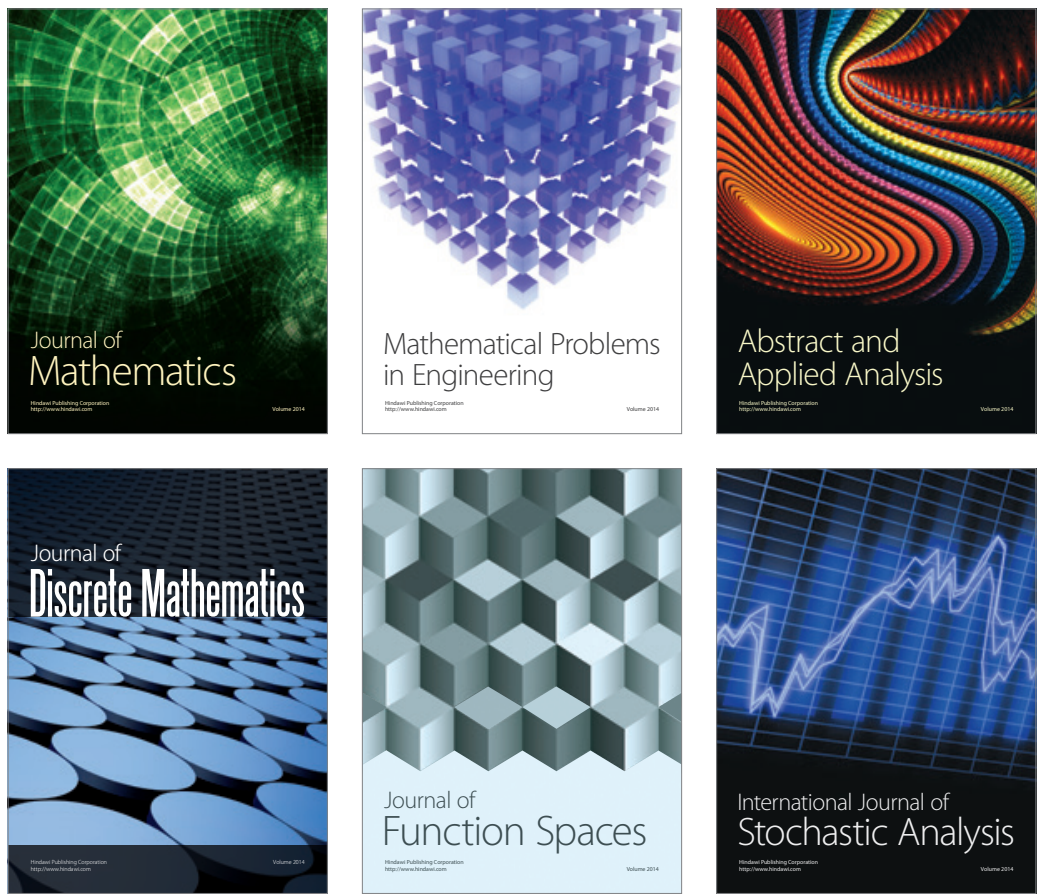

Journal of

Function Spaces

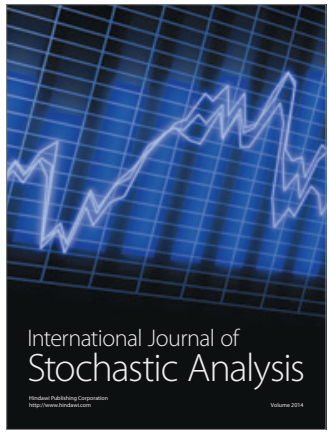

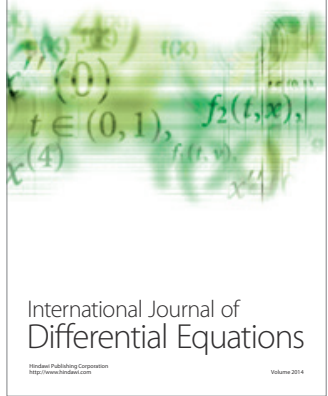
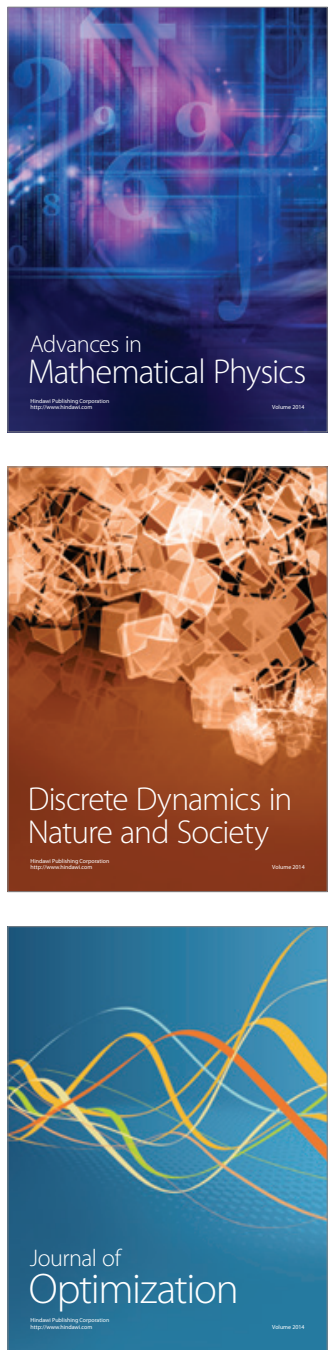